\title{
The Clinical Value of Serum Adropin Level in Early Detection of Diabetic Nephropathy
}

\author{
Ali Es-haghia ${ }^{\text {Tuqa Al-Abyadh }}{ }^{a}$ Hassan Mehrad-Majd ${ }^{b}$

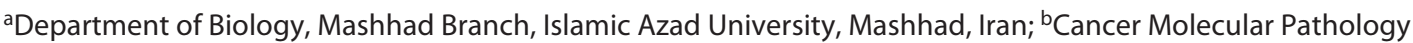 \\ Research Center, Mashhad University of Medical Sciences, Mashhad, Iran
}

\section{Keywords}

Adropin · Type 2 diabetes · Nephropathy · Glomerular filtration rate

\begin{abstract}
Background/Aims: Adropin is a metabolic hormone secreted by the liver, brain, and many peripheral tissues and is involved in energy homeostasis and insulin sensitivity. Some reports have indicated a significant decrease in serum adropin levels in type 2 diabetic patients. However, the significance of a decline in adropin level in early detection of diabetic nephropathy (DN) remains to be clarified. The purpose of this study was to evaluate the serum levels of adropin in patients with type 2 diabetes with and without nephropathy. Methods: A total of 135 unrelated subjects (including 45 diabetic patients with nephropathy, 45 without nephropathy, and 45 healthy controls) were enrolled in this study. Fasting venous blood samples were collected from all patients. Serum adropin levels of all cases were analyzed by an enzymelinked immunosorbent assay method. The correlations of serum adropin levels with anthropometric and biochemistry variables were determined. Logistic regression was performed to assess the association of adropin with odds of nephropathy. A receiver operating characteristic (ROC) curve was obtained to explore the optimum serum adropin concentration in distinguishing diabetic patients with and without nephropathy. Results: Diabetic patients with nephropa-
\end{abstract}

karger@karger.com www.karger.com/kbr

Karger"

GOPEN ACCESS
(C) 2021 The Author(s)

Published by S. Karger AG, Basel

This is an Open Access article licensed under the Creative Common Attribution-NonCommercial-4.0 International License (CC BY-NC) (http://www.karger.com/Services/OpenAccessLicense), applicable to the online version of the article only. Usage and distribution for commercial purposes requires written permission. thy showed lower serum adropin levels than those in patients without nephropathy and healthy controls $(p<0.001)$. Pearson correlation analysis indicated that serum adropin was negatively correlated with $\mathrm{BMI}, \mathrm{FBS}, \mathrm{HbA} 1 \mathrm{c}$, blood urea, creatinine, $L D L$, and $A C R$ and positively correlated with $H D L$ and albumin. Logistic regression analysis showed that serum adropin was correlated with decreased risk of developing diabetic nephropathy. Moreover, in ROC analysis, at cutoff value $3.20(\mathrm{mg} / \mathrm{dL})$ with an AUC $=0.830$, adropin had $80 \%$ sensitivity and $60 \%$ specificity for distinguishing the diabetic nephropathy. Conclusions: This study demonstrates that decreased level of adropin is associated with renal dysfunction in patients with type 2 diabetes mellitus. Serum adropin concentrations may be used as a biomarker for early detection of diabetic nephropathy.

(c) 2021 The Author(s).

Published by S. Karger AG, Basel

\section{Introduction}

Diabetes is one of the most common metabolic diseases associated with chronic hyperglycemia and impaired carbohydrate, fat, and protein metabolism [1]. Millions of people worldwide have the disease, and it is estimated that the number of diabetics will reach 300 million by 2025 [2]. Complications such as cardiovascular disease, peripheral vascular involvement, blindness, strokes, skin diseases, and orthopedic and mental disorders are associated with 
diabetes [3]. Meanwhile, one of the late complications of diabetes is diabetic nephropathy.

Diabetic nephropathy is one of the predisposing factors for chronic kidney disease [4]. It is characterized by persistent albuminuria (excretion of albumin $>300 \mathrm{mg} / \mathrm{h}$ in $24 \mathrm{~h}$ or $>200 \mu \mathrm{g} / \mathrm{min}$ ) or a ratio of $\mathrm{Alb} / \mathrm{Cr}>20$ in the morning urine along with other features such as the presence of diabetic retinopathy, and lack of other laboratory or clinical findings may indicate other renal diseases [5, 6]. Albumin excretion rate and glomerular filtration rate (GFR) are the 2 main criteria for the diagnosis and staging of diabetic nephropathy and microalbuminuria as its first clinical sign $[5,7]$.

Although microalbuminuria (primary nephropathy) is one of the important predictors of progression to proteinuria $(>300 \mathrm{mg} / \mathrm{dL})$ or nephropathy, these measurements are not useful enough for early detection and monitoring of the disease progression $[8,9]$. In some patients, especially patients with type 2 diabetes, despite lack of albumin excretion, chronic kidney disease has also been observed, indicating the incompatibility of albuminuria for the monitoring of renal nephropathy [8]. This inconsistency has been reported in several cases. For example, some patients with microalbuminuria have normal renal function while some cases of diabetic patients with normoalbuminuria have nephrotic renal disorders $[10,11]$. This inconsistency and invalidity of serum creatinine for early detection of renal dysfunction strongly justifies the need for new high potential biomarkers for early detection of diabetic nephropathy, especially in type 2 diabetes patients. Adropin is a 76-amino acid peptide that has been shown to be correlated with energy regulation and obesity [12]. Animal intervention studies have reported the effective role of adropin in lowering blood glucose and improving insulin resistance in diabetic mice [13]. Also, a few studies investigating the serum level of adropin in diabetic patients in comparison with healthy individuals have shown a decreased level of adropin in diabetic patients [14].

Although some animal studies indicated the role of adropin in the pathogenesis of diabetic nephropathy, there have not been many human studies considering the possible association of serum adropin levels with diabetic nephropathy. The existing data support the idea that adropin may be involved in the progression and development of diabetic nephropathy [15]. Considering the evidence mentioned above, the current study was designed to investigate the serum adropin levels in Iraqi diabetic patients with and without nephropathy and also to elucidate possible application of adropin levels for early detection of nephropathy in patients with type 2 diabetes.

\section{Materials and Methods}

\section{Subjects}

A total of 135 unrelated Iraqi subjects (including 45 diabetic patients with nephropathy, 45 without nephropathy, and 45 healthy controls) were enrolled in this study. All patients attending in diabetic clinics of Al-Zahra hospital, Iraq, were selected consecutively between October 2015 and December 2018. The patients were all diagnosed with T2DM in accordance with the American Diabetic Association criteria [16]. Urine albumin to creatinine ratio (ACR) was used to categorize T2DM patients into 2 groups of diabetes with and without nephropathy. Accordingly, patients with ACR $<30 \mathrm{mg} / \mathrm{g}$ were assigned as normoalbuminuric diabetics (diabetic patients without nephropathy), while diabetic patients with ACR $\geq 30 \mathrm{mg} / \mathrm{g}$ were defined as hyperalbuminurics (diabetic patients with nephropathy). After reading and signing the informed consent form, all participants attended a complete clinical examination and fasting venous blood sample collection. Subjects were excluded from the study if they had acute infectious disease during the past week, active immunologic disease, history of cardiovascular disease, history of uncontrolled hypertension, cancer, and any other endocrine diseases. Healthy controls were selected from the subjects who had undergone the general health check-up and had no history of diabetes, medication, or dietary supplements.

\section{Biochemical Measurements}

For each patient, a total of $10 \mathrm{~mL}$ of venous blood sample was taken in the morning after at least $12 \mathrm{~h}$ of fasting. After clotting the blood, serum was extracted by centrifugation within $3 \mathrm{~h}$ and stored at $-80^{\circ} \mathrm{C}$ until analysis. Biochemical parameters including lowdensity lipoprotein (LDL), high-density lipoprotein (HDL), VLDL (very-low-density lipoprotein), triglyceride (TG), total cholesterol (TC), serum creatinine, and total protein were measured according to international standard procedures using Roche Diagnostics Reagents for Cobas ${ }^{\mathrm{TM}} \mathrm{C} 111$ Chemistry Analyzer (Roche Diagnostics, Indianapolis, IN, USA). Fasting blood glucose was immediately measured by a glucose oxidase procedure. Glycosylated hemoglobin A1c (HbAlc) was measured using cation-exchange column chromatography on an automatic analyzer. Serum adropin levels of all cases were analyzed by an enzyme-linked immunosorbent assay method according to the manufacturer's instructions.

\section{Statistical Analysis}

IBM SPSS Statistics 22 was used for all of the statistical analyses, and $p$ value $<0.05$ was considered significant. Continuous variables were expressed as mean $\pm S D$, and categorical data were presented as number or percentage. Student's $t$ test was used for normally distributed variables to compare the differences between 2 groups, while one-way ANOVA was used for multiple comparisons. The $\chi^{2}$ test was used to compare categorical data. The association between serum adropin levels and metabolic parameters was measured using Pearson's correlation analysis. Logistic regression was performed to assess the association of adropin with odds of nephropathy. The receiver operating characteristic (ROC) curve was obtained to explore the area under the curve and optimum serum adropin concentration for distinguishing diabetic nephropathy. The optimal cutpoint value is defined as a concentration in which sensitivity and specificity are the closest to the value of the area under the ROC curve (the point closest to corner $[0,1]$ in the ROC plane) [17]. 
Table 1. Demographic and clinical characteristics of T2DM patients with and without nephropathy and healthy controls

\begin{tabular}{|c|c|c|c|c|c|}
\hline Gender (M/F) & $21 / 23$ & $23 / 22$ & ns & $22 / 23$ & ns \\
\hline Age, years & $51.43 \pm 6.04$ & $47.60 \pm 4.87$ & 0.003 & $48.67 \pm 5.08$ & 0.042 \\
\hline $\mathrm{HbA} 1 \mathrm{c}, \%$ & $8.44 \pm 0.76$ & $7.90 \pm 0.45$ & $<0.001$ & $4.94 \pm 0.28$ & $<0.001$ \\
\hline $\mathrm{FBS}, \mathrm{mg} / \mathrm{dL}$ & $216.61 \pm 70.54$ & $220.98 \pm 43.24$ & 0.903 & $96.88 \pm 11.51$ & $<0.001$ \\
\hline $\mathrm{HDL}-\mathrm{C}, \mathrm{mg} / \mathrm{dL}$ & $42.33 \pm 4.76$ & $42.98 \pm 6.07$ & 0.857 & $54.24 \pm 6.15$ & $<0.001$ \\
\hline LDL-C, mg/dL & $120.25 \pm 17.84$ & $114.30 \pm 12.25$ & 0.102 & $111.55 \pm 9.57$ & 0.009 \\
\hline VLDL, mg/dL & $29.64 \pm 7.32$ & $27.97 \pm 8.17$ & 0.537 & $29.22 \pm 6.60$ & 0.962 \\
\hline $\mathrm{TG}, \mathrm{mg} / \mathrm{dL}$ & $148.35 \pm 36.58$ & $139.95 \pm 40.87$ & 0.533 & $146.22 \pm 32.95$ & 0.960 \\
\hline Albumin, g/dL & $3.31 \pm 0.32$ & $4.09 \pm 0.49$ & $<0.001$ & $4.39 \pm 0.66$ & $<0.001$ \\
\hline Urea, mg/dL & $46.34 \pm 4.57$ & $33.95 \pm 4.42$ & $<0.001$ & $31.09 \pm 4.45$ & $<0.001$ \\
\hline Adropin, ng/mL & $2.77 \pm 0.36$ & $3.35 \pm 0.36$ & $<0.001$ & $4.21 \pm 0.52$ & $<0.001$ \\
\hline
\end{tabular}

Values were expressed as mean \pm SD. eGFR is calculated by the abbreviated MDRD equation: $186 \times(\mathrm{creatinine} / 88.4)^{-1.154} \times(\mathrm{age})^{-0.203}$ $\times(0.742$ if female $) \times(1.210$ if black). DM, diabetes mellitus; HbA1c, hemoglobin A1c; FBS, fasting blood sugar; TC, total cholesterol; HDL-C, high-density lipoprotein cholesterol; LDL-C, low-density lipoprotein cholesterol; VLDL, very-low-density lipoprotein cholesterol; TG, triglycerides; T-protein, total protein; ACR, albumin to creatinine ratio; eGFR, estimated glomerular filtration rate; ns, nonsignificant.

\section{Results}

\section{Baseline Clinical Characteristics of T2DM Patients and Controls}

In the present study, a total of 45 cases with diabetic nephropathy, 45 without nephropathy, and 45 healthy controls were included. Demographic, clinical, and biochemical data of diabetic patients with and without nephropathy as well as healthy controls are presented in Table 1 . Subjects in the diabetic nephropathy group had higher levels of $\mathrm{HbAlc}$, blood urea, creatinine, and ACR and lower levels of eGFR and albumin compared with those without nephropathy and healthy control group.

\section{Serum Adropin Concentrations}

As shown in Table 1, serum adropin levels were significantly reduced in diabetic patients with nephropathy compared with 2 other groups $(p<0.001)$. A significant difference was also found for serum adropin level between type 2 diabetic patients without nephropathy and healthy control group $(p<0.001)$. As shown in Figure 1, it is evident that the degree of decrease in serum adropin levels is increased from healthy individuals toward the diabetic patients with nephropathy.
Correlation between Serum Adropin Levels and Other Clinical Characteristics

Correlation analysis between serum adropin levels and various demographic and clinical parameters such as age, FBS, HbA1c, duration of diabetes, urea, creatinine, TC, TG, HDL, VLDL, LDL, total protein, ACR, and eGFR was performed in this study. As shown in Table 2, serum adropin levels were inversely correlated with FBS, HbA1c, urea, creatinine, LDL, and ACR. Moreover, a significant direct correlation was found regarding HDL, eGFR, and albumin (Fig. 2A, B). However, no significant correlation was observed between serum adropin levels and age, duration of diabetes, VLDL, and total protein.

\section{The Association of Serum Adropin Concentrations} with Diabetic Nephropathy

In order to evaluate the clinical value of serum adropin level in predicting the diabetic nephropathy, logistic regression analysis was performed both in crude and adjusted for age and gender variables (Table 3 ). The results showed that serum adropin level is significantly associated with the risk of diabetic nephropathy development $(\mathrm{OR}=0.014,95 \% \mathrm{CI}=0.002-0.084, p<0.001)$. This strong negative correlation still remained significant even after adjusting for age and gender $(\mathrm{OR}=0.012,95 \% \mathrm{CI}=$ 
Fig. 1. Serum adropin levels in diabetic patients with and without nephropathy and healthy control groups.

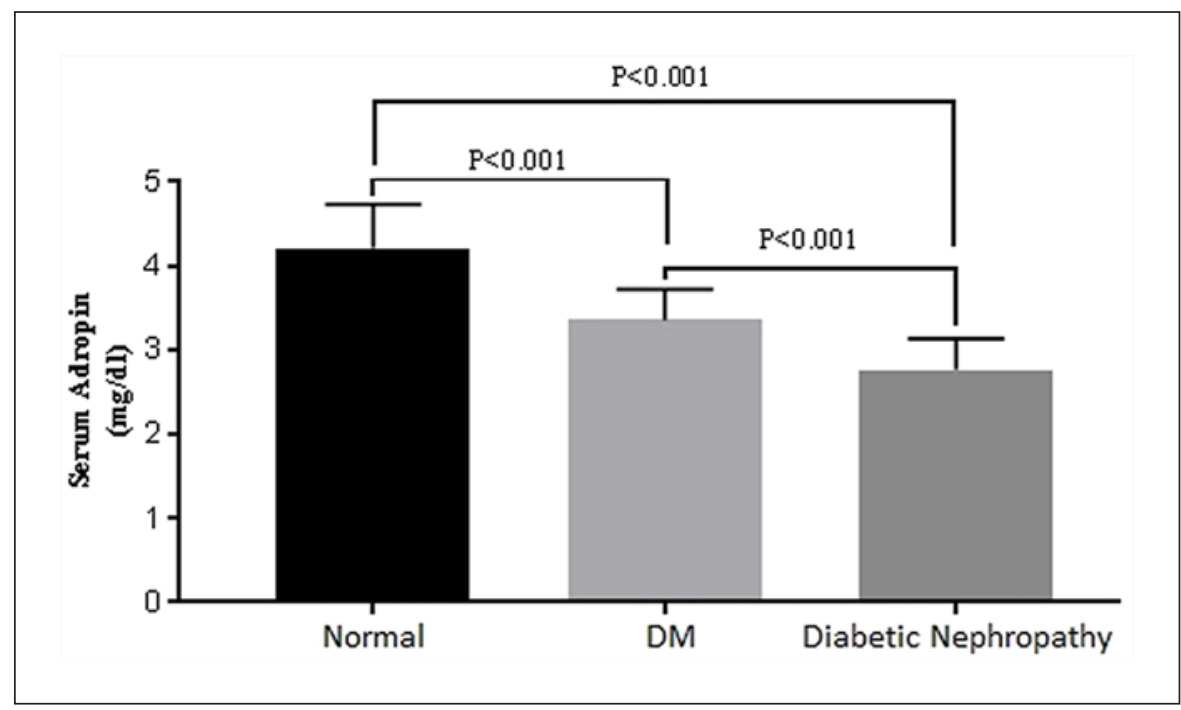

Table 2. Correlations between adropin with demographic and clinical characteristics

\begin{tabular}{llr}
\hline Adropin variables & $r$ & $p$ value \\
\hline Age & -0.120 & 0.168 \\
FBS & -0.530 & $<0.001$ \\
HbA1c & -0.743 & $<0.001$ \\
DM duration & -0.171 & 0.108 \\
Urea & -0.628 & $<0.001$ \\
Creatinine & -0.250 & 0.004 \\
Cholesterol & 0.002 & 0.984 \\
TG & -0.078 & 0.370 \\
HDL & 0.498 & $<0.001$ \\
VLDL & -0.078 & 0.373 \\
LDL & -0.207 & 0.016 \\
Albumin & 0.513 & $<0.001$ \\
T-protein & -0.116 & 0.182 \\
ACR & -0.711 & $<0.001$ \\
eGFR & 0.190 & 0.031 \\
\hline
\end{tabular}

FBS, fasting blood sugar; $\mathrm{HbA} 1 \mathrm{c}$, hemoglobin A1 c; DM, diabetes mellitus; TG, triglycerides; HDL-C, high-density lipoprotein cholesterol; VLDL, very-low-density lipoprotein cholesterol; LDL-C, low-density lipoprotein cholesterol; T-protein, total protein; ACR, albumin to creatinine ratio.

$0.002-0.83, p<0.001)$. Furthermore, univariate logistic regression revealed that along with adropin other factors including age, $\mathrm{HbA1c}$, DM duration, albumin, urea, creatinine, ACR, and eGFR showed a significant association $(p<0.05)$ with nephropathy (Table 3$)$. When considering together in the backward stepwise multivariate logistic regression model, adropin remained as a significant inde- pendent predictor of diabetic nephropathy $(\mathrm{OR}=0.011$, $95 \% \mathrm{CI}=0.01-0.43, p=0.016$ ).

\section{ROC Analyses of Adropin Levels}

The ROC curve analyses have been performed to evaluate if adropin could be a novel biomarker for distinguishing diabetic nephropathy form those without nephropathy and healthy control groups. The ROC analysis results are shown in Figure 3. According to the ROC curve in the present study, the optimal cutoff value of adropin to differentiate diabetic nephropathy and nondiabetic nephropathy was $3.20(\mathrm{mg} / \mathrm{dL})$, with a sensitivity of $80 \%$, specificity $60 \%$, and area under the curve $=0.830$.

\section{Discussion}

In the present study, the results showed that serum adropin levels were significantly reduced in diabetic patients with nephropathy compared with diabetic patients without nephropathy and healthy nondiabetic individuals. Also, a significant inverse correlation was observed between serum adropin level and metabolic-related parameters, which indicates a possible protective role of adropin against the development of metabolic disorders, especially diabetes. The findings of this study demonstrate that decreased level of adropin is associated with renal dysfunctions in patients with type 2 diabetes mellitus and could be used as a biomarker in early detection of diabetes nephropathy.

Adropin has been introduced as a potential regulatory hormone in energy hemostasis, maintaining insulin sen- 


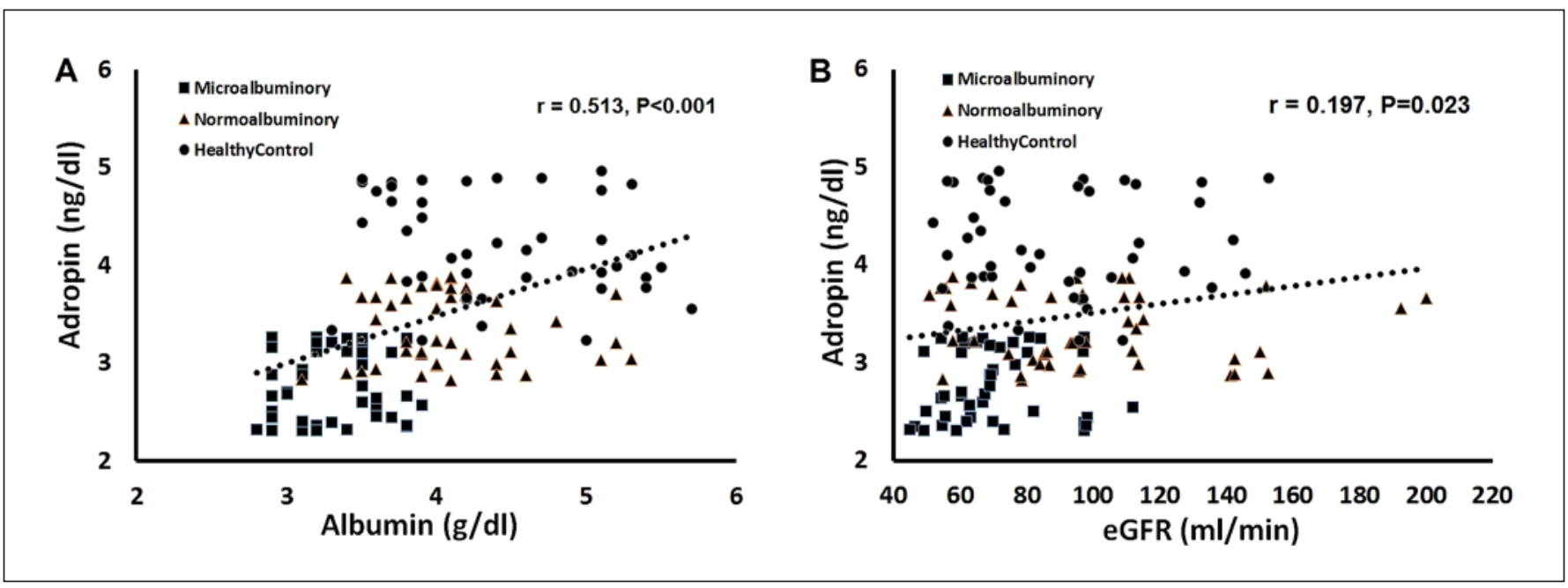

Fig. 2. The scatter plots displaying the correlation between serum levels of adropin with albumin (A) and eGFR (B). eGFR, estimated glomerular filtration rate.

Table 3. Logistic regression analysis for determining the factors associated with diabetic nephropathy

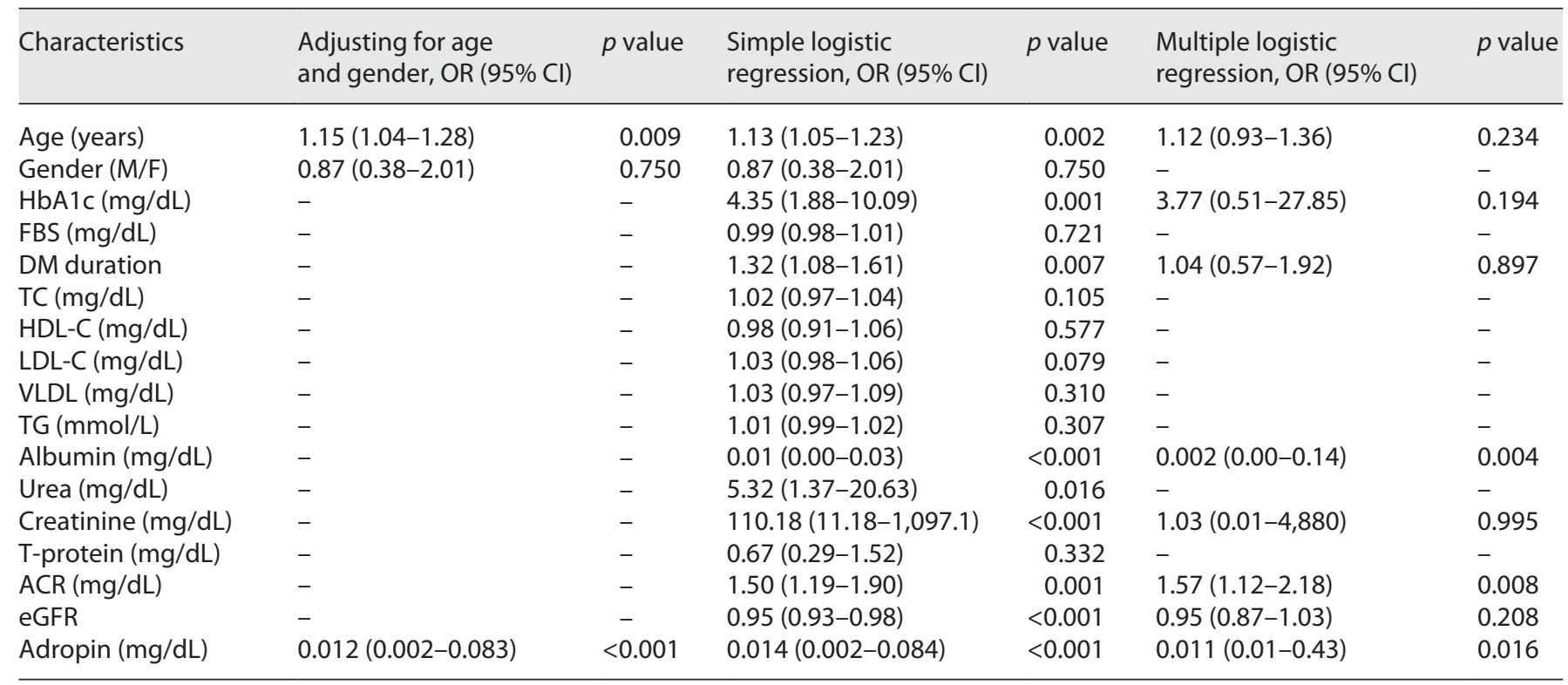

FBS, fasting blood sugar; HbA1c, hemoglobin A1c; DM, diabetes mellitus; TG, triglycerides; HDL-C, high-density lipoprotein cholesterol; VLDL, very-low-density lipoprotein cholesterol; LDL-C, low-density lipoprotein cholesterol; T-protein, total protein; ACR, albumin to creatinine ratio.

sitivity and glucose tolerance in mice $[18,19]$. It has been shown that adropin treatment in streptozotocin-induced diabetic rats has improved glucose tolerance, enhanced insulin sensitivity, and reduced insulin resistance [20]. Besides, adropin knockout mice have shown severe insulin resistance, adiposity, and dyslipidemia. In addition to animal model studies, few human-based research studies were also conducted to further explore the possible correlation of serum adropin levels with metabolic disorders.

In one study by Celik et al. [21], it was shown that adropin level in maternal and cord serum of the gestational diabetes mellitus (GDM) patients was significantly 


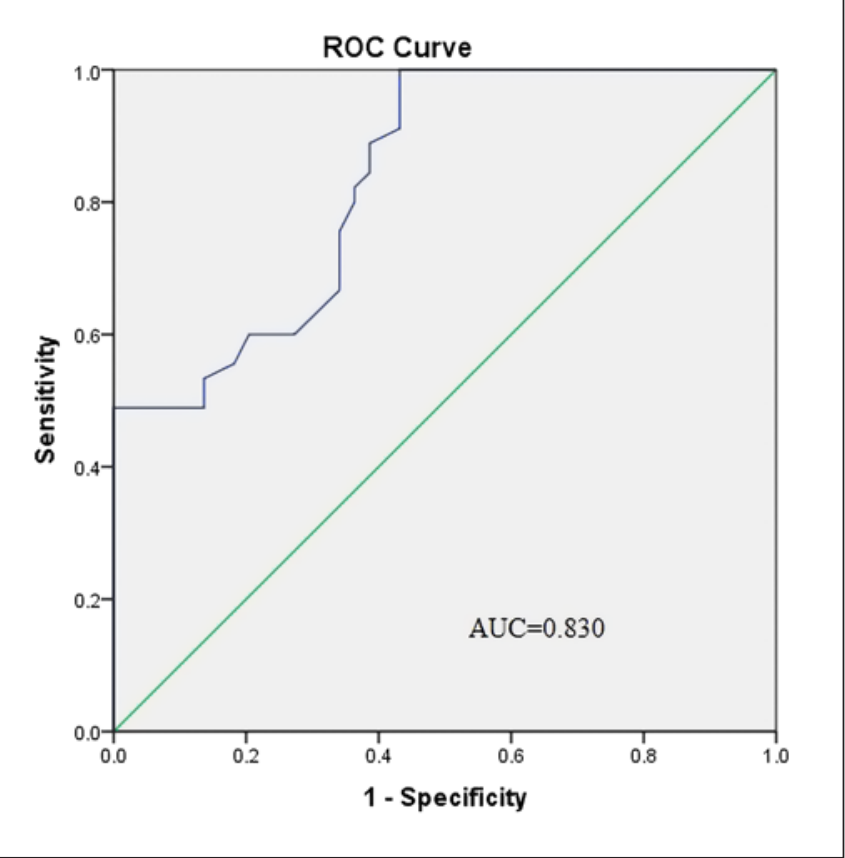

Fig. 3. The ROC curve for determining the adropin cutoff value for identifying diabetic nephropathy. ROC, receiver operating characteristic.

associated with the development of GDM. Compared with the healthy controls, serum adropin levels were significantly lower in patients with GDM [22]. In another study conducted by Zang et al. [14], serum adropin concentrations were decreased in Chinese T2DM patients, especially in overweight/obese subjects. The same results were also found by $\mathrm{Wu}$ et al. [23] which reported a significant reduced serum adropin level in T2DM patients than in nondiabetic patients. They have shown low serum adropin was also associated with coronary atherosclerosis in T2DM and nondiabetic patients.

Up to now, only 1 study was found to investigate the potential of adropin level in development of diabetic nephropathy [24]. Hu and Chen [24] have shown that serum adropin levels were negatively associated with renal function and may be implicated in the pathogenesis of diabetic nephropathy development. Our results were consistent with findings by $\mathrm{Hu}$ and Chen [24]. We found that T2DM patients with nephropathy exhibited relatively lower adropin levels than those without nephropathy. However, in contrast to the previous study, in the present study, we found a negative correlation of serum adropin levels with HbAlc which is a parameter for average blood glucose. Therefore, regarding this discrepancy, further studies in different populations are necessary to clarify this possible association.
Our logistic regression analysis demonstrated that serum adropin level was correlated with decreased risk of developing diabetes retinopathy. This indicates that serum adropin could serve as a biomarker for predicting the risk of developing diabetic nephropathy. In some studies, adropin expression was detected in the kidney tissue of rats [13]. Accordingly, increased adropin immunoreaction was seen in the kidney of diabetes-induced rats compared to control subjects [13]. It has also been demonstrated that the intensities of adropin immunoreactivity increased with diabetic severity [25]. However, serum adropin levels have been reported to be lower in those conditions that require further investigation to elucidate this discrepancy. In agreement with these findings, our results showed a decreasing trend for serum adropin level from healthy controls toward diabetes nephropathy.

This study has some limitations. First, due to the cross-sectional design of the study, it is not clear whether adropin reduction has led to development of nephropathy or vice versa. Hence, future longitudinal studies are needed to shed light on the causative relation. Second, adropin concentrations were only measured at fasting conditions, which could not reflect adropin fluctuation over time especially after the different dietary content. Considering the kinetics of adropin clearance from the circulation through measuring its concentration in various conditions may help to clarify the possible mechanisms involved in the response of adropin to altered metabolic status. Finally, multicentered studies, with relatively higher sample sizes, are warranted to confirm our results.

\section{Conclusions}

The present study revealed that serum adropin, with a significantly different level in diabetic patients, is associated with renal dysfunction indicating a potential role in the pathogenesis of diabetic nephropathy development and early detection of renal dysfunction. Further molecular and genetic studies are required to clarify the largely unknown aspects of adropin in the pathophysiology of diabetic nephropathy.

\section{Acknowledgments}

We would like to thank all the study participants for their cooperation. We would also like to thank the Clinical Research Development Center, Ghaem Hospital, Mashhad University of Medical Sciences, for their assistance in this manuscript. 


\section{Statement of Ethics}

The study protocol was approved by the Research Ethics Committee of Mashhad University of Medical Sciences (Ref. No. IR.MUMS.MEDICAL.REC.1399.812). All subjects have given their written informed consent.

\section{Conflict of Interest Statement}

The authors declare no conflicts of interest regarding the publication of this manuscript.

\section{Funding Sources}

This work was supported by Research Project No. 991472 as MSc dissertation, financed by the Mashhad University of Medical Sciences.

\section{Author Contributions}

H. Mehrad-Majd and A. Es-haghi contributed to study concept and design, T. Al-Abyadh contributed to acquisition of data, $\mathrm{H}$. Mehrad-Majd contributed to statistical analysis, T. Al-Abyadh and H. Mehrad-Majd contributed to drafting of the manuscript, and A. Es-haghi contributed to study supervision.

\section{Data Availability Statement}

All data generated or analyzed during this study are included in this article. Further enquiries can be directed to the corresponding author.

\section{References}

1 American Diabetes Association. Diagnosis and classification of diabetes mellitus. Diabetes Care. 2005;28(Suppl 1):S37-42.

2 Björk S. The cost of diabetes and diabetes care. Diabetes Res Clin Pract. 2001;54:13-8.

3 Deshpande AD, Harris-Hayes M, Schootman M. Epidemiology of diabetes and diabetes-related complications. Phys Ther. 2008;88(11): 1254-64.

4 John S. Complication in diabetic nephropathy. Diabetes Metab Syndr. 2016;10(4):247-9.

5 Viberti GC, Hill RD, Jarrett RJ, Argyropoulos A, Mahmud U, Keen H. Microalbuminuria as a predictor of clinical nephropathy in insulindependent diabetes mellitus. Lancet. 1982; 1(8287):1430-2.

6 Schena FP, Gesualdo L. Pathogenetic mechanisms of diabetic nephropathy. J Am Soc Nephrol. 2005;16(3 Suppl 1):S30-3.

7 Mogensen CE, Christensen CK. Predicting diabetic nephropathy in insulin-dependent patients. N Engl J Med. 1984;311(2):89-93.

8 Newman D, Mattock M, Dawnay A, Kerry S, McGuire A, Yaqoob M, et al. Systematic review on urine albumin testing for early detection of diabetic complications. Health Technol Assess. 2005;9(30):iii-vi, xiii-163.

9 Levey AS, Becker C, Inker LA. Glomerular filtration rate and albuminuria for detection and staging of acute and chronic kidney disease in adults: a systematic review. JAMA. 2015;313(8):837-46.

10 Lin CH, Chang YC, Chuang LM. Early detection of diabetic kidney disease: present limitations and future perspectives. World J Diabetes. 2016;7(14):290-301.
11 Persson F, Rossing P. Diagnosis of diabetic kidney disease: state of the art and future perspective. Kidney Int Suppl. 2018;8(1):2-7.

12 Kumar KG, Trevaskis JL, Lam DD, Sutton GM, Koza RA, Chouljenko VN, et al. Identification of adropin as a secreted factor linking dietary macronutrient intake with energy homeostasis and lipid metabolism. Cell Metab. 2008 Dec;8(6):468-81.

13 Aydin S, Kuloglu T, Aydin S, Eren MN, Yilmaz M, Kalayci M, et al. Expression of adropin in rat brain, cerebellum, kidneys, heart, liver, and pancreas in streptozotocininduced diabetes. Mol Cell Biochem. 2013 Aug;380(1-2):73-81.

14 Zang H, Jiang F, Cheng X, Xu H, Hu X. Serum adropin levels are decreased in Chinese type 2 diabetic patients and negatively correlated with body mass index. Endocr J. 2018 Jul 28; 65(7):685-91.

$15 \mathrm{Hu}$ W, Chen L. Association of serum adropin concentrations with diabetic nephropathy. Mediators Inflamm. 2016;2016:6038261.

16 American Diabetes Association. Diagnosis and classification of diabetes mellitus. Diabetes Care. 2014 Jan;37(Suppl 1):S81-90.

17 Unal I. Defining an optimal cut-point value in ROC analysis: an alternative approach. Comput Math Methods Med. 2017;2017: 3762651.

18 Kumar KG, Trevaskis JL, Lam DD, Sutton GM, Koza RA, Chouljenko VN, et al. Identification of adropin as a secreted factor linking dietary macronutrient intake with energy homeostasis and lipid metabolism. Cell Metab. 2008;8(6):468-81.
19 Ganesh Kumar K, Zhang J, Gao S, Rossi J, McGuinness OP, Halem $\mathrm{HH}$, et al. Adropin deficiency is associated with increased adiposity and insulin resistance. Obesity. 2012;20(7): 1394-402.

20 Gao S, McMillan RP, Zhu Q, Lopaschuk GD, Hulver MW, Butler AA. Therapeutic effects of adropin on glucose tolerance and substrate utilization in diet-induced obese mice with insulin resistance. Mol Metab. 2015;4(4):310-24.

21 Celik E, Yilmaz E, Celik O, Ulas M, Turkcuoglu I, Karaer A, et al. Maternal and fetal adropin levels in gestational diabetes mellitus. Perinat Med. 2013;41(4):375-80.

22 Beigi A, Shirzad N, Nikpour F, Nasli Esfahani E, Emamgholipour S, Bandarian F. Association between serum adropin levels and gestational diabetes mellitus; a case-control study. Gynecol Endocrinol. 2015;31(12):939-41.

23 Wu L, Fang J, Chen L, Zhao Z, Luo Y, Lin C, et al. Low serum adropin is associated with coronary atherosclerosis in type 2 diabetic and non-diabetic patients. Clin Chem Lab Med. 2014;52(5):751-8.

$24 \mathrm{Hu} \mathrm{W}$, Chen L. Association of serum adropin concentrations with diabetic nephropathy. Mediators inflamm. 2016;2016:6038261.

25 Kuloğlu T, Aydin S. Immunohistochemical expressions of adropin and inducible nitricoxide synthase in renal tissues of rats with streptozotocin-induced experimental diabetes. Biotech Histochem. 2014;89(2):104-10. 